\title{
Sirtuin Activator and Inhibitor Affect Early Dictyostelium Development upon Starvation
}

\author{
Shuhei Soeda, Hideo Taniura* \\ Laboratory of Neurochemistry, College of Pharmaceutical Sciences, Ritsumeikan University, Shiga, Japan \\ Email address: \\ Soeda05 $a$ fc.ritsumei.ac.jp (S. Shuhei), hideo $a$ fc.ritsumei.ac.jp (H. Taniura) \\ ${ }^{*}$ Corresponding author
}

To cite this article:

Shuhei Soeda, Hideo Taniura. Sirtuin Activator and Inhibitor Affect Early Dictyostelium Development upon Starvation. Cell Biology. Vol. 6, No. 1, 2018, pp. 13-19. doi: 10.11648/j.cb.20180601.13

Received: June 3, 2018; Accepted: July 5, 2018; Published: August 2, 2018

\begin{abstract}
Sirtuin is an evolutionally conserved histone deacetylase. The effects of sirtuin activator, resveratrol and inhibitor, nicotinamide on early Dictyostelium development upon starvation were examined. The cell streaming was appeared at $7 \mathrm{~h}$ and tight aggregates was formed at $24 \mathrm{~h}$ in control cells under submerged culture. Resveratrol treatment accelerated the development. Cell streaming at $6 \mathrm{~h}$ and weak aggregates at $9 \mathrm{~h}$ were already observed. While, nicotinamide treatment delayed the development. The initiation of streaming was delayed at $9 \mathrm{~h}$, and the development was still under a cell streaming even after 24 h. Dictyostelium development starts inducing the essential molecules adenylyl cyclase, aca and cAMP pulsing induces cell-surface cAMP receptor (carA). Resveratrol treatment significantly increased the expression level of aca at 4 and $6 \mathrm{~h}$ after starvation. Nicotinamide treatment decreased the aca expression level at $4 \mathrm{~h}$ and carA expression level at $6 \mathrm{~h}$. The induction of aca upon starvation starts with the transcriptional activation by MybB, a. putative transcription factor. MybB expression recovered the initiation timing of the cell streaming against the effect of nicotinamide, but the aggregates formation was still impaired at $24 \mathrm{~h}$. Resveratrol had no effects on MybB-null mutant cells. Neither streaming nor aggregates formation was observed even after $24 \mathrm{~h}$ with low levels of aca expression after starvation by resveratrol treatment. These findings suggest that sirtuins may participate in more than one point of the early developmental processes of Dictyostelium upon starvation.
\end{abstract}

Keywords: Sirtuin, Resveratrol, Nicotinamide, Cellular Development, Dictyostelium discoideum

\section{Introduction}

The first known sirtuin, Sir2 (silent information regulator 2) of Saccharomyces cerevisiae regulates ribosomal DNA recombination, gene silencing, DNA repair, chromosomal stability and longevity $[1,2]$. Sirtuins have been defined as a family of nicotinamide adenine dinucleotide (NAD)dependent enzymes that deacetylate lysine residue on various proteins. It has been recognized that growing number of non-histone proteins are deacetylated by situins. Sirtuin deacetylates a target protein, and acetyl group from the target protein is transferred to the ADP-ribose moiety of NAD. Certain sirtuins have in addition an ADPribosyltransferase activity. In mammals, seven homologs, Sirt1-Sirt7, have been identified. Sirt1 is a best studied mammalian NAD-dependent histone deacetylase that downregulate the acetylation levels of many regulatory proteins including p53, FOXO, PPAP $\gamma$, and PGC- $1 \alpha$ involved in energy homeostasis, DNA repair, cell survival and lifespan extension $[3,4]$.

The cellular slime mold Dictyostelium discoideum is an excellent model organism for studying the molecular basis of cell differentiation and signal transduction. Although Dictyostelium cells grow vegetatively as individual amebae, upon starvation they gather with neighboring cells forming aggregates consisting of up to $10^{5}$ cells. Aggregation is mediated by the chemotaxis of cells toward cAMP to form a multicellular aggregate. During this process, cells can be seen streaming toward a central domain of aggregation center. After aggregation, cells behave as in more complex multicellular organisms and finally culminate into a fruiting body consisting of spores on top of a supporting stalk [5]. 
cAMP levels are controlled by the developmentally regulated expression of adenylate cyclase, aca. The aca is expressed from the first hours of development. cAMP is used both as a secreted intercellular signal and as an intracellular second messenger in signal transduction. Binding of cAMP to cAMP receptor, carA activates several signaling pathways. Some of them activate the chemotactic movement of the cells $[6,7]$. The sirtuins are conserved throughout evolution from archaebacterial to eukaryotes. Dictyostelium discoideum encodes at least 5 sirtuin proteins (Sir2A-E) that show sequence similarity to human sirtuins [8]. Sirt1 activator, resveratrol (RSV), and inhibitor, nicotinamide (NAM), are commonly used for studying Sirt1 function $[9,10,11]$. Here, the effects of RSV and NAM on Dictyostelium development upon starvation were investigated.

\section{Materials and Methods}

\subsection{Growth and Development of Dictyostelium}

Dictyostelium discoideum Ax-2 cells were provided by the NBPR (National BioResources Project) of Japan. Ax-2 cells were grown axenically in HL5 medium, and for development under submerged culture, the cells were washed twice in 10 $\mathrm{mmol} / \mathrm{L} \mathrm{Na-K}$ phosphate buffer $(10 \mathrm{mmol} / \mathrm{L} \mathrm{Na}-\mathrm{K}$ phosphate buffer, $20 \mathrm{mmol} / \mathrm{L} \mathrm{MgSO}_{4}$ and $2 \mathrm{mmol} / \mathrm{L} \mathrm{CaCl}_{2}, \mathrm{pH}$ 6.1) and the cells at $2 \times 10^{6}$ cells $/ \mathrm{mL}$ in Na-K buffer was developed over $24 \mathrm{~h}$ [12]. At a day before the experiment, resveratrol (RSV) (Wako, Japan) at $50 \mu \mathrm{mol} / \mathrm{L}$ or nicotinamide (NAM) (Wako, Japan) at $10 \mathrm{mmol} / \mathrm{L}$ was added in axenic growth medium HL5 and the cells were developed in the presence of RSV or NAM at the same concentration. RSV was dissolved in methanol, and NAM in water. RSV at $0 \mu \mathrm{mol} / \mathrm{L}$ (methanol) or NAM at $0 \mathrm{mmol} / \mathrm{L}$ (water) was used as a control.

\begin{tabular}{|c|c|c|}
\hline Sir2A & 209 & GVGRGPKIVAPSEQEESEEDESCVLKKPTIIEEIAKYINS- \\
\hline $\operatorname{Sir} 2 \mathrm{C}$ & 167 & - \\
\hline Sir2D & 226 & IDPIEFTKDIGFKLELEKDDDAWEIITAFLTRKKVAVNL---FLNYLKYNTLARPYRKKI \\
\hline SIRT1 & 204 & PPKRKKRK-DINTIEDAVKL- \\
\hline Sir2A & 248 & -AKCKNIIVMTGAGISVAAGIPDFRSPKTGLYP-EKLDKYDLPYRE \\
\hline Sir2C & 178 & DKCKNIIVLTGAGISVASGIPDFRSVETGLYNNENVSKFKLPFKE \\
\hline Sir2D & 283 & ATLDLSTFEKVCQLFESSKNIVIITGAGVSVSCGIPDFRS-KGGVYETIEKKY-NLPRPE \\
\hline SIRT1 & 242 & - LQECKRIIV_TGAGVSVSCGIPDFRS-RDGIVARLAVDFPDLPDPQ \\
\hline Sir2A & 291 & AIFDIEYYFKKNPKPFYYLLSKELFP-OSFNPTTVFYFIKLLSDKGLLRNETQNIDTLERI \\
\hline Sir2C & 223 & ANFDIDYFKFNPEPFYQLSKDL YPSGKFKCTPVHYY IKLL SDKGL LRNYARNADTLERI \\
\hline Sir2D & 341 & SLFDI HYRANPL LFFEFAKEIFP-GNHKPSPTHSFIKLLDEKGKLLRNYTQNIDTLEHV \\
\hline SIRT1 & 287 & AMEDIEYFRKDPRPFFKFAKEIYP-GRFDPSLCEKEIAL SDKEGKLLRNYTQNIDTLERV \\
\hline Sir2A & 350 & AGIPANKLVEAHGSFATSHCVSCKKEYSTEYVKER IFKDE-----LPECTETSGCKGI-- \\
\hline Sir2C & 283 & AGIPLDKLIEAHGSFAVSRCINCCGLEYSQEYIKDSIIFNNDPLKSVVPRCKVVQCNNAV-- \\
\hline Sir2D & 400 & AGIDREKLVNCHGSFSTATCITCKL TVDGTTIRDTIMKME- - - IPLLCRQCNDGQSF-- \\
\hline SIRT1 & 346 & AGIRR--ILQCHGSFATASCLICKYKVDCEEA \\
\hline Sir2A & 403 & VKPDIVFFGESLPSRFNDCAREDFTKCDLLLVIGTSLKVHPFASLINF- -AKKCPRVILI \\
\hline Sir2C & 341 & IKPDIVFFGESLPPIFNQNILDDINRCDCL IVIGISLKVDPIASMVHFFPHFKNIPRLLI \\
\hline Sir2D & 453 & MKPDIVFFGENLPDRFDQCVLKDVKDIDLLIVMGSSLDVDPVSLLPDIVD--KRIPRILI \\
\hline SIRT1 & 399 & MKPEIVFFGENLPEQF HRAMKYDKDEVDLLIVIGSSLKVRPVAL IPSSIP--HEVPRILI \\
\hline Sir2A & 460 & 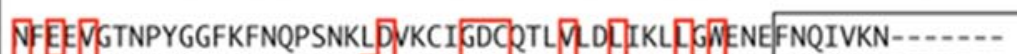 \\
\hline Sir2C & 401 & NNQIVGENSFGGFNFNN-NKNFDFKMIGDCQESVINLSKLLNWDTELLLNLINSKNHN--- \\
\hline Sir2D & 511 & NREL VAQP - - \\
\hline SIRT1 & 457 & NREPLPHLH - ......... FDVLLLDCDVIINELCHREGGEYAKLCCNPVKLSEITE \\
\hline
\end{tabular}

Figure 1. Amino acid sequence alignment of mouse Sirt1 catalytic domain with Sir2A, Sir2C and Sir2D derived from Dictyostelium discoideum.

Amino acid sequences of around murine Sirt1 catalytic domain (NCBI NM_019812.3), Sir2A (DDB_G0283917), Sir2C (DDB_G0284795) and Sir2D (DDB_G0289967) were aligned using ClustalW. Boxed area in black is the catalytic domain in sirtuin. Identical residues among the sequences are boxed in red. Amino acid residue numbers are shown at each line. 


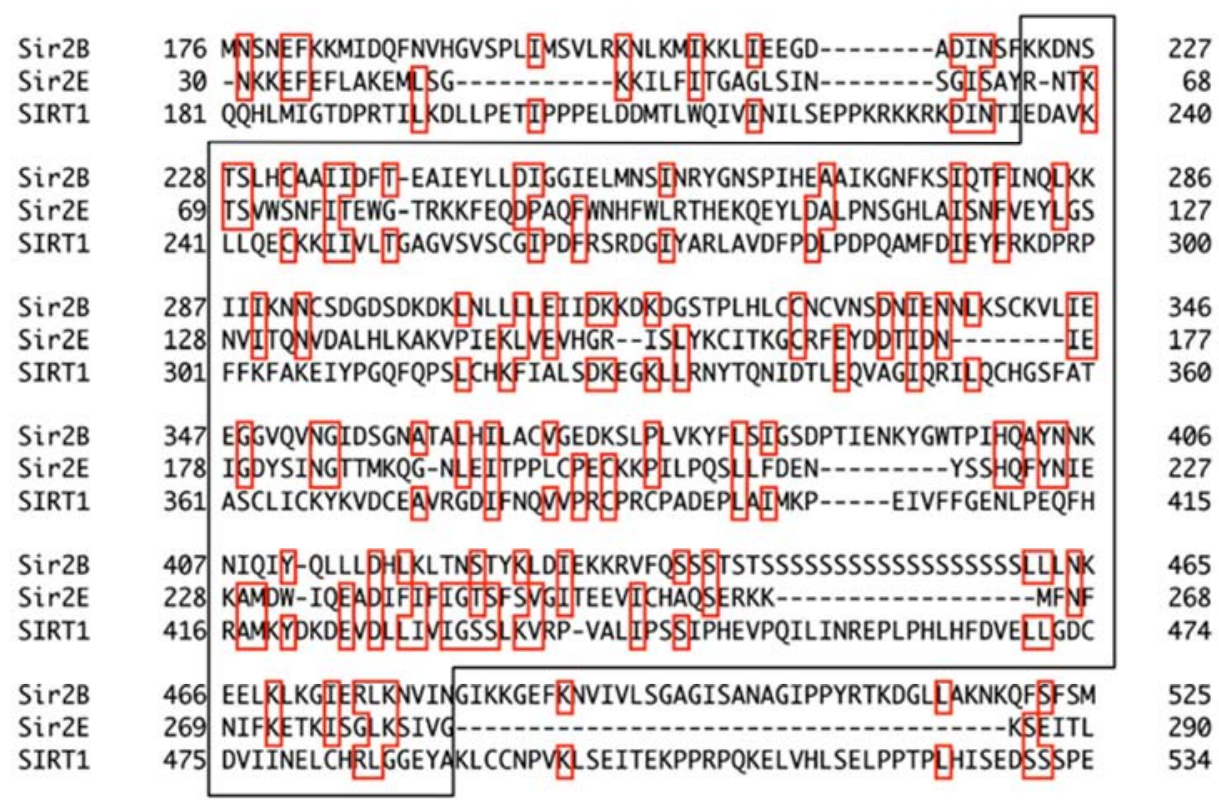

Figure 2. Amino acid sequence alignment of mouse Sirt1 catalytic domain with Sir2B and Sir2E derived from Dictyostelium discoideum.

Amino acid sequences of around murine Sirtl catalytic domain (NCBI NM_019812.3), Sir2B (DDB_G0286671), and Sir2E (DDB_G0270928) were aligned using ClustalW. Boxed area in black is the catalytic domain in sirtuin. Identical residues among the sequences are boxed in red. Amino acid residue numbers are shown at each line.

\subsection{Quantitative RT-PCR ( $q R T-P C R)$}

Total RNA was extracted from vegetative $(0 \mathrm{~h})$ and 2, 4 and 6 h-starved Ax-2 cells in the presence or absence of RSV or NAM under submerged culture using TRI reagent (SigmaAldrich), and cDNA was synthesized from total RNA (1 $\mu \mathrm{g})$. cDNA was used as templates for PCR. RT-PCR products were quantified with LightCycler FastStart DNA Master-PLUS SYBR Green I (Roche Diagnostics) in a LightCycler instrument (Rche Diagnostics). Melting curves were analyzed to confirm a single species of each PCR product. Actin 15 cDNA was used as an internal standard to quantify the relative expression of each cDNA. Statistical significance was tested using Student's t-test. Primer used for real-time PCR are as follows: Actin15 (forward, 5'- taaatccaaaagccaacagag-3'; reverse, 5'-ttggaaagttgagagtgaagc-3'); aca (forward, 5'ttgcaggtttccaagaata-3'; reverse, 5'-tttgctctactgataccgat-3'); carA (forward, 5'-taaatatgtttccaccagca-3'; recerse, 5'taccacgacttgaactatatg-3').

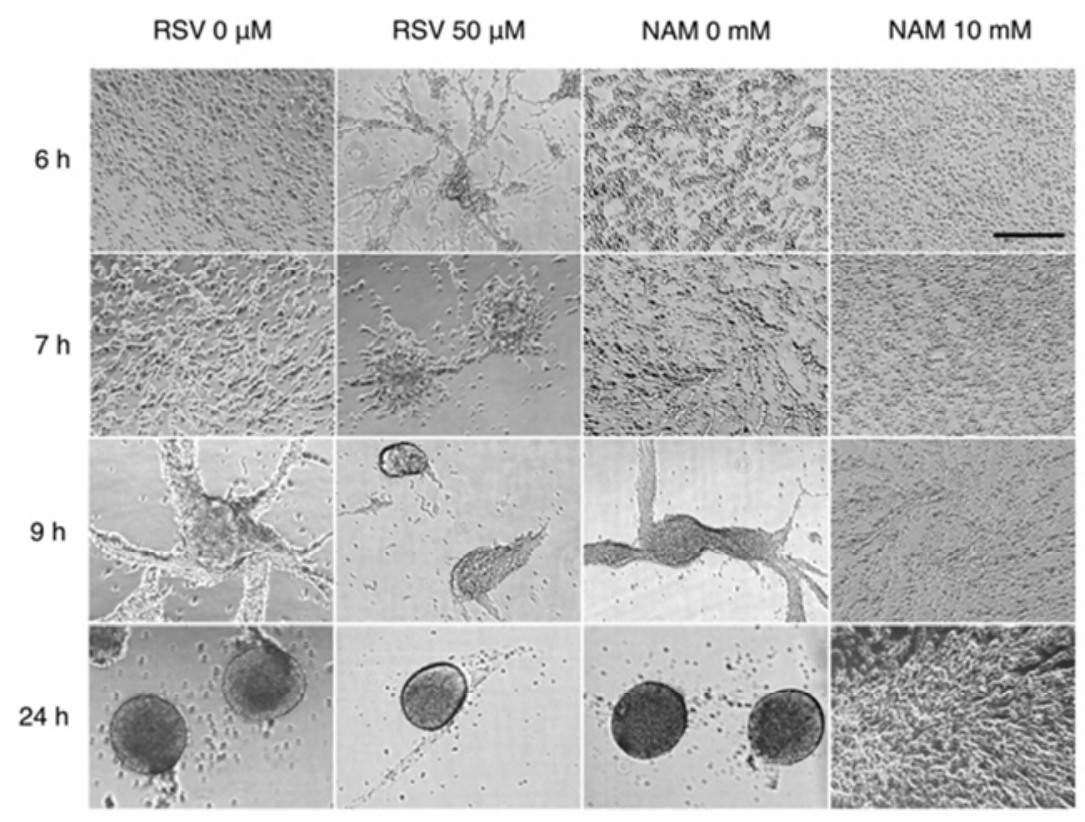

Figure 3. Effect of RSV and NAM on cellular development. 


\subsection{Cloning and Expression of MybB}

The entire coding sequence of MybB (DDB_G275445) was amplified from cDNA and cloned into pBluescript SK+ using BamHI and XhoI sites. The following primers were used for PCR amplification: forward, 5'-tgcggatccatgactgctatattcccaaat3'; reverse, 5'-gcgctcgagatagtaataattttacagcat-3'. The mybB sequence was matched completely to the sequence in the Dictybase. To express GFP-tagged MybB, mybB cDNA was cloned into pTX-GFP expression vector. GFP tag was fused at the $\mathrm{N}$ terminus of MybB. The construct was introduced into cells by electroporation and stable transformants were selected with G418. For fluorescence immunocytochemistry, cells were fixed in $4 \%$ formaldehyde in phosphate buffered saline (PBS) for $20 \mathrm{~min}$ and incubated with the anti-GFP rabbit monoclonal antibody (Life Technologies). For DAPI (Life Technologies) staining, the fixed cells were treated with $300 \mathrm{nmol} / \mathrm{L}$ DAPI in PBS for $10 \mathrm{~min}$ at room temperature and observed using a fluorescence microscope (EVOS, Thermo Fisher). Growth and development of GFP-MybB expressing cells in the absence or presence of NAM was the same as described in 2.1.

Ax-2 cells were developed at $2 \times 10^{6}$ cells $/ \mathrm{mL}$ under submerged culture in the absence or presence of RSV (50 $\mu \mathrm{M})$ or NAM $(10 \mathrm{mM})$. RSV at $0 \mu \mathrm{mol} / \mathrm{L}$ (methanol) or $\mathrm{NAM}$ at $0 \mathrm{mmol} / \mathrm{L}$ (water) was used as a control. Development was imaged at 6, 7, 9 and $24 \mathrm{~h}$ after starvation. Scale bar: $200 \mu \mathrm{m}$.

\subsection{Isolation of MybB-null Mutant}

The MybB-null mutant was generated by gene targeting according to the method described previously [6]. The targeting construct contains a genomic fragment that encompasses the MybB coding region from the position +32 to $+2949(+1$ at the beginning of the ATG start codon) in which a BSR cassette was inserted at the SacI site (the position +1501) (Figure 6A). The MybB gene was amplified by PCR using primers containing PstI and EcoRI sites. The following primers were used for PCR amplification: forward, 5'-tgcctgcagcattttattattgggctagca-3'; reverse, 5'gcggaattcaatattgataagatgggaaat-3'. The construct was linearized with PstI and EcoRI and introduced into Ax-2 cells. Transformants were selected for blasticidin resistance.

Ax-2 cells were developed at $2 \times 10^{6}$ cells $/ \mathrm{mL}$ under submerged culture in the absence (Control) or presence of RSV $(50 \mu \mathrm{M})$ or NAM $(10 \mathrm{mM})$. Total RNA was extracted from the cells at $0,2,4$ and $6 \mathrm{~h}$ after starvation. qRT-PCR was performed using specific primers for aca, carA or actin15. The quantified results were normalized against actin15 mRNA levels. Each value represents the mean \pm standard error $(\mathrm{SE})(\mathrm{n}=3)$. Asterisk indicates a value that significantly differ $\left(* \mathrm{P}<0.05,{ }^{* *} \mathrm{P}<0.0001\right)$ from the value of the control.

\section{Results}

The sirtuin family of protein contains the highly conserved catalytic core domain composed of a large oxidized NADbinding Rossmann fold subunit [13]. When mouse Sirt1 catalytic domain sequence (amino acids 236-490) was compared with Dictyostelium sirtuin sequences, Sir2A, Sir2C and Sir2D contained highly homologous sequences (Figure 1), but not with Sir2B and Sir2E. Sequence similarities showed more than $50 \%$ identity between mouse Sirt1 and Dictyostelium Sir2A, Sir2C or Sir2D. While, about $10 \%$ identity between mouse Sirt1 and Sir2B or Sir2E was shown in Figure 2. Thus, Sir2A, Sir2C and Sir2D may have a deacetylase enzyme activity and could be a target for the Sirt1 activator, RSV and inhibitor, NAM.

Dictyostelium discoideum Ax-2 cells were grown axenically in HL-5 medium [12] and developed under submerged culture over $24 \mathrm{~h}$ in the presence or absence of RSV or NAM. As shown in Figure 3, the cell streaming was appeared at $7 \mathrm{~h}$ and tight aggregates was formed at $24 \mathrm{~h}$ in control cells. The Sirt1 activator RSV treatment accelerated the development. Cell streaming at $6 \mathrm{~h}$ and weak aggregates at $9 \mathrm{~h}$ were already observed. While, the Sirt1 inhibitor NAM treatment suppressed the development. The initiation of streaming was delayed at $9 \mathrm{~h}$, and the development was still under a cell streaming even after $24 \mathrm{~h}$. The cell streaming was gradually condensing to form aggregates seen in control cells at $9 \mathrm{~h}$. But condensing the streaming was severely impaired by NAM treatment at $24 \mathrm{~h}$.

Development in Dictyostelium upon starvation starts inducing the essential molecules to produce and sense extracellular cAMP such as an adenylyl cyclase (aca) and cell-surface cAMP receptor (carA) [6,14]. Aca is the key enzyme of the cAMP signaling. Thus, aca mRNA expression levels in the cells treated with either RSV or NAM during the early development were examined by qRT-PCR. As shown in Figure $4 \mathrm{~A}$ and $\mathrm{B}$, aca was induced upon starvation and RSV treatment significantly increased the expression level at 4 and $6 \mathrm{~h}$ after starvation by $1.9 \mathrm{x}$ and $2.3 \mathrm{x}$ fold, respectively (Figure 4A). NAM treatment significantly reduced the aca expression level at $4 \mathrm{~h}$ to $29 \%$ compared with that of control cells (Figure 4B), suggesting that the reduction of aca expression at $4 \mathrm{~h}$ is why NAM treatment delayed the initiation of cell streaming. NAM treatment also significantly reduced carA expression level at $6 \mathrm{~h}$ to $25 \%$ compared with that of control cells (Figure 4C).

A. Expression of GFP-tagged MybB. GFP-tagged MybB was detected using anti-GFP antibody in axenically grown Ax-2 cells under a fluorescence microscope (middle panel, $\alpha$ GFP). The same cells were stained with DAPI to show the nuclei (right panel, DAPI). The left panel shows the phase contrast image of the same field of cells (Phase). Scale bar: $50 \mu \mathrm{m}$.

B. Effect of RSV on cellular development of MybB OE cells. Control and MybB OE (GFP-MybB) cells were developed at $2 \times 10^{6}$ cells $/ \mathrm{mL}$ under submerged culture. MybB OE cells in the presence of NAM $(10 \mathrm{mM})$ were developed under the same condition as described. Development was imaged at 5,8 and $24 \mathrm{~h}$ after starvation. Scale bar: $200 \mu \mathrm{m}$. 


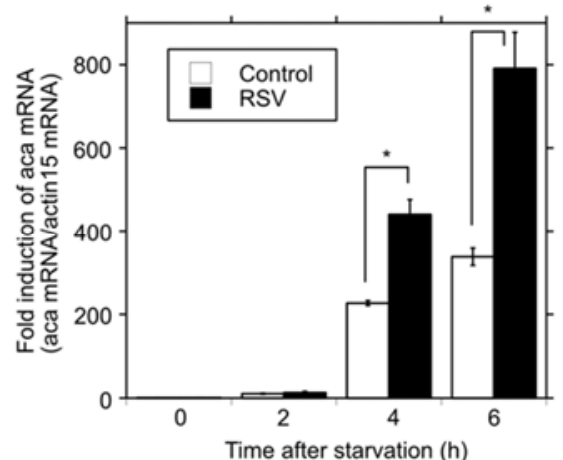

B

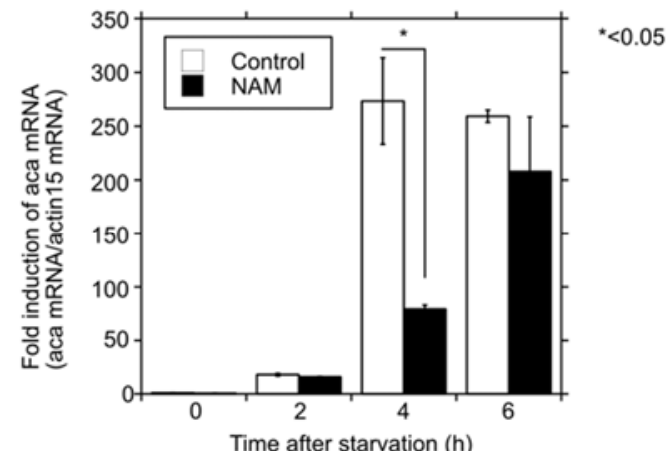

C

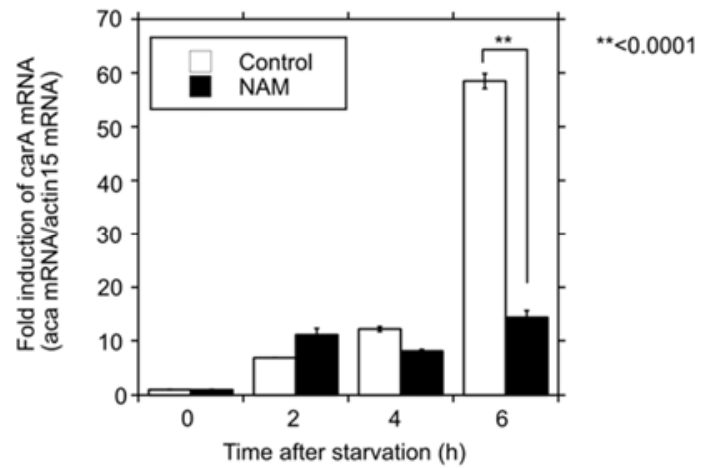

Figure 4. Alteration of $m R N A$ expression levels of aca ( $A$ and $B$ ) and carA (C) by RSV (A) or NAM (B and C).

C. mRNA expression levels of aca. Control cells, MybB $\mathrm{OE}$ cells and MybB OE cells treated with NAM were developed under submerged culture. Total RNA was extracted from the cells at $0,2,4$ and $6 \mathrm{~h}$ after starvation. qRT-PCR was performed using specific primers for aca or actin15. The quantified results were normalized against actin15 mRNA levels. Each value represents the mean \pm standard error $(\mathrm{SE})(\mathrm{n}=3)$. Asterisk indicates a value that significantly differ $(* \mathrm{P}<0.05)$ from the value of the control.

MybB is a homologue of the Myb-related transcription factor in Dictyostelium and has been identified as being one of the components regulating aca expression at the growth/differentiation transition $[6,15,16]$. When MybB was expressed as GFP tagged protein at the $\mathrm{N}$ terminus (MybB OE cells), the GFP-MybB immunoreactivity was relatively enriched in nuclei (Figure 5A). GFP-MybB expression accelerated the development. Cell streaming was observed at $5 \mathrm{~h}$ accompanied by significant increased expression of aca at 2 and $4 \mathrm{~h}$ compared to the levels of control cells (Figure 5B and C). When MybB OE cells were treated with NAM, the timing of cell streaming was recovered comparable to the development of control cells against NAM treatment (Figure 5B and C compare to Figure 3). However, the aggregates formation of MybB OE cells was severely impaired at $24 \mathrm{~h}$ (Figure 5B). The result suggests that MybB expression is not sufficient for restoring the developmental impairment by NAM. Thus, NAM also inhibits later developmental process during the aggregates formation. Next, MybB-null mutant was isolated according to the construct containing a genomic fragment that encompasses the MybB coding region from the position +32 to $+2949(+1$ at the beginning of the ATG start codon) in which a BSR cassette was inserted at the SacI site (the position +1501) (Figure 6A). When MybB-null mutant cells were starved, they displayed an aggregate-less phenotype even after $24 \mathrm{~h}$ (Figure 6B). The Sirt1 activator RSV had no effects on MybB-null mutant cells. Neither streaming nor aggregates formation was observed even after $24 \mathrm{~h}$ by RSV treatment (Figure 6B). MybB-null mutant cells and the mutant cells treated with RSV showed quite low levels of aca expression after starvation (Figure 6C). Thus, the effect of RSV on development requires MybB.

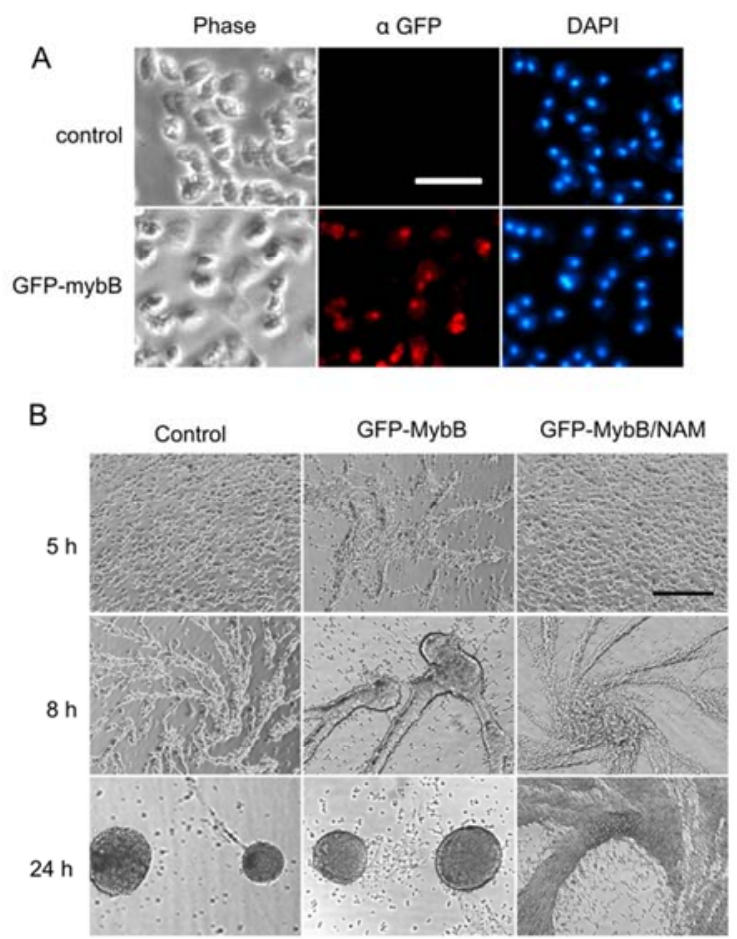

C

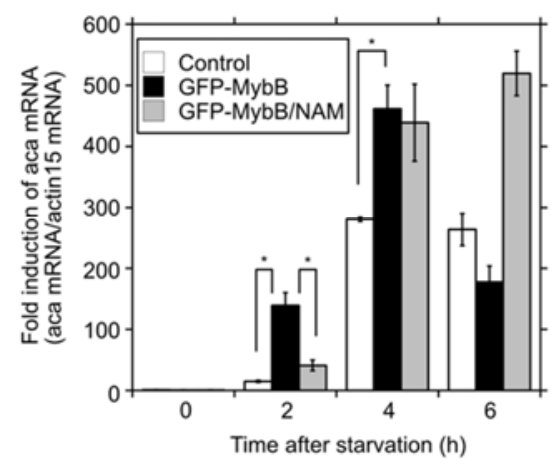

Figure 5. Effect of RSV on cellular development of MybB OE cells. 
A

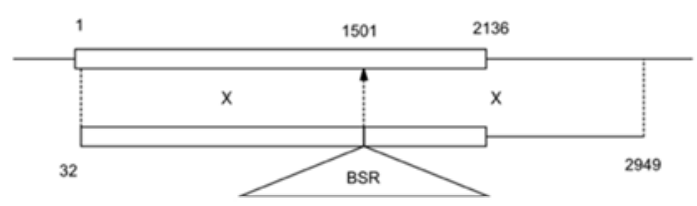

B

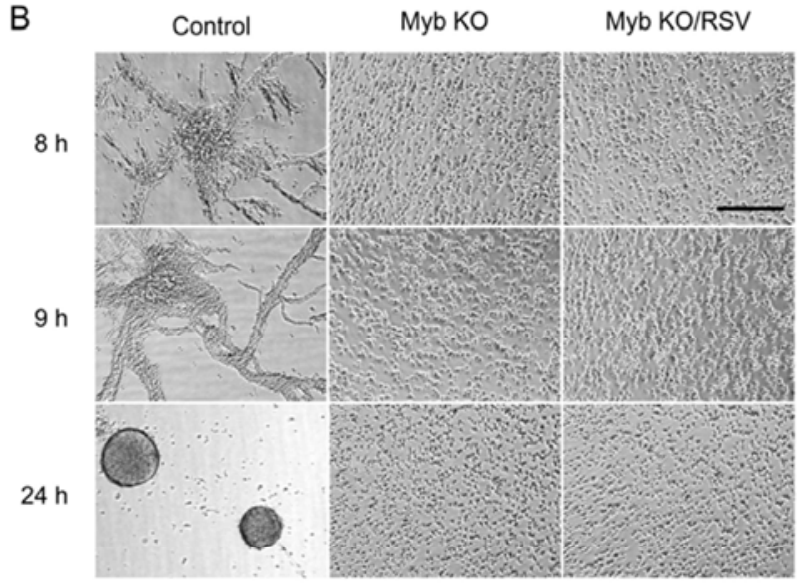

C

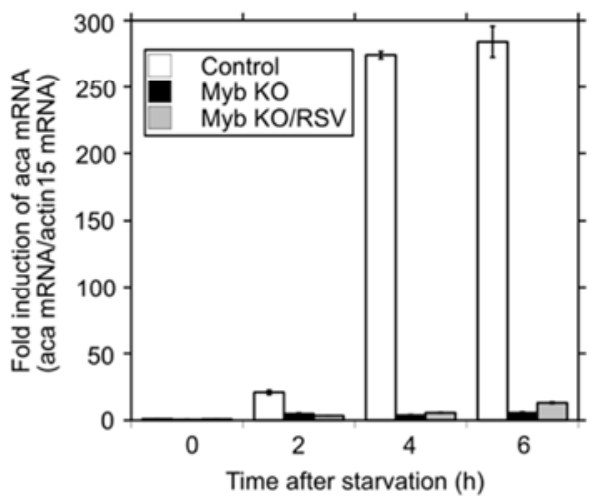

Figure 6. Effect of RSV on MybB-null mutant cells.

A. MybB-null mutant construction. The targeting construct contains a genomic fragment that encompasses the MybB coding region from the position +32 to +2949 ( +1 at the beginning of the ATG start codon) in which a BSR cassette was inserted at the SacI site (the position +1501).

B. Effect of RSV on cellular development of MybB-null mutant. Control and MybB-null mutant (MybB KO) cells were developed at $2 \times 10^{6}$ cells $/ \mathrm{mL}$ under submerged culture. MybB-null mutant cells in the presence of RSV $(50 \mu \mathrm{M})$ were developed under the same condition as described. Development was imaged at 8,9 and $24 \mathrm{~h}$ after starvation. Scale bar: $200 \mu \mathrm{m}$.

C. mRNA expression levels of aca. Control cells, MybBnull mutant cells and MybB-null mutant cells treated with RSV were developed under submerged culture. Total RNA was extracted from the cells at $0,2,4$ and $6 \mathrm{~h}$ after starvation. qRT-PCR was performed using specific primers for aca or actin 15. The quantified results were normalized against actin15 mRNA levels. Each value represents the mean \pm standard error $(\mathrm{SE})(\mathrm{n}=3)$.

\section{Discussion}

The sirtuin family of protein is highly conserved from prokaryotes to human. Sir2A, Sir2C and Sir2D among 5 sirtuins in Dictyostelium contains highly homologous catalytic domain sequences to mammalian Sirt1, suggesting that these sirtuins function as similar fashion with Sirt1. Sirt1 is involved in energy homeostasis through the interaction with several transcription factors including p53, FOXO, PPAP $\gamma$ and PGC- $1 \alpha$. Upon starvation, Sirt1 binds and represses genes controlled by PPAP $\gamma$, including genes mediating fat storage, to mobilize fat in white adipocytes. RSV and NAM are widely used for studying Sirt1 function $[5,6,7]$. Sirt1 activator RSV accelerated the development accompanied by an increased the aca expression upon starvation. While, Sirt1 inhibitor NAM delayed the initiation of streaming with reduction of the initial aca induction. The results suggest that sirtuins in Dictyostelium are involved in the early development upon starvation. The induction of aca upon starvation starts with the transcriptional activation by MybB, a putative transcription factor [3]. Because RSV had no effects on MybB-null mutant cells, one of the Dictyostelium sirtuin among Sir2A, Sir2C or Sir2D may interact and regulate the MybB activity. CarA is another important molecule for the streaming and aggregate formation. Transcription of carA from the early, aggregation promoter is activated by cAMP pulsing $[10,11]$. Developmental timing of the cell streaming was recovered in MybB OE cells against NAM treatment, but the aggregates formation was still impaired at $24 \mathrm{~h}$. These findings suggest that sirtuins may participate in more than one points of the early developmental processes of Dictyostelium upon starvation.

\section{Conclusion}

Sirt 1 activator RSV and inhibitor NAM affect the early Dictyostelium development upon starvation. Identification of the surtuins involved in the early development and its target molecule are necessary to confirm the sirtuins function in Dictyostelium discoideum.

\section{References}

[1] S. Imai, C. M. Armstrong, M. Kaeberlein, and L. Guarente. "Transcriptional silencing and longevity protein Sir2 is an NAD-dependent histone deacetylase," 2000, Nature 403, 795800 .

[2] J. Landry, A. Sutton, S. T. Tafrov, R. C. Heller, J. Stebbins, L. Pillus, and R. Sternglanz. "The silencing protein SIR2 and its homologs are NAD-dependent protein deacetylases," 2000, Proc. Natl. Acad. Sci. USA 97, 5807-5811.

[3] H. Yamamoto, K. Schoonjans, and J. Auwerx. "Sirtuin functions in health and disease," Mol. Endoclinol. 2007, 21, 1745-1755.

[4] S. Michan, and D. Sinclair. "Sirtuins in mammals: insights into their biological function," 2007, Biochem. J. 404, 1-13. 
[5] P. Schaap. "Evolution of developmental signaling in Dictyostelid social amoebas," 2016, Curr. Opin. Genet. Dev. 39, 29-34.

[6] H. Otsuka, and P. J. M. Van Haastert. "A novel Myb homolog initiates Dictyostelium development by induction of adenylyl cyclase expression," 1998, Genes Develop. 12, 1738-1748.

[7] J. Rodriguez-Centeno, and L. Sastre. "Biological activity of the alternative promoters of the Dictyostelium discoideum adenylyl cyclase A gene,” 2016, PLOS ONE 0148533.

[8] T. Katayama, and H. Yasukawa. "Analysis of Sir2E in the cellular slime mold Dictyostelium discoideum: Cellular localization, spatial expression and overexpression," 2008, Develop. Growth. Differ. 50, 645-652.

[9] F. Picard, M. Kurtev, N. Chung, A. Topark-Ngram, T. Senewong, R. M. de Oliveira, M. Leid, M. W. McBurney, and L. Guarente. "Sirt1 promotes fat mobilization in white adipocytes by repressing PPAR- $\gamma$," 2004, Nature 429, 771777 .

[10] A. Brunet, L. B. Sweeney, J. F. Sturgill, K. F. Chua, P. L. Greer, Y. Lin, H. Tran, S. E. Ross, R. Mostoslavsky, H. Y. Cohen, L. S. Hu, H-L. Cheng, M. P. Jedrychowski, S. P. Gygi, D. A. Sinclair, F. W. Alt, and M. E. Greenberg. "Stressdependent regulation of FOXO transcription factor by the SIRT1 deacetylase," 2004, Science 303, 2011-2015.
[11] S. Nemoto, M. M. Fergusson, and T. Finkel. "SIRT1 functionally interacts with metabolic regulator and transcriptional coactivator PGC-1 $\alpha, 2005$, J. Biol. Chem. 280, 16456-16460.

[12] H. Taniura, N. Tanabe, Y. Bando, and N. Arai. "Nse1 and Nse4, subunits of the Smc5-Smc6 complex, are involved in Dictyostelium development upon starvation," 2015, Develop. Growth. Differ. 57, 430-443.

[13] A. Vassilopoulos, K. S. Fritz, D. R. Petersen, and D. Gius. "The human sirtuin family: Evolutionary divergences and functions," 2011, Human Genomics 5, 485-496.

[14] T. J. Sun, and P. N. Devreotes. "Gene targeting of the aggregation stage cAMP receptor cAR1 in Dictyostelium," 1991, Genes Develop. 5, 572-582.

[15] X. Mu, S. A. Spanos, J. Shiloach, and A. Kimmel. "CRTF is a novel transcription factor that regulate multiple stages of Dictyostelium development." 2001, Development 128, 25692579.

[16] T. Kon, H. Adachi, and K. Sutoh. "amiB, a novel gene required for the growth/differentiation transition in Dictyostelium," 2000, Genes to Cells 5, 43-55. 